ISSN 1996-1944

www.mdpi.com/journal/materials

Article

\title{
Magnetization Dynamics of Amorphous Ribbons and Wires Studied by Inductance Spectroscopy
}

\section{Israel Betancourt}

Departamento de Materiales Metálicos y Cerámicos, Instituto de Investigaciones en Materiales, Universidad Nacional Autónoma de México, D.F. 04510, Mexico; E-Mail: israelb@ correo.unam.mx; Tel.: +52-55-56224641; Fax: +52-55-56161371

Received: 5 November 2010; in revised form: 23 November 2010 / Accepted: 6 December 2010 / Published: 23 December 2010

\begin{abstract}
Inductance spectroscopy is a particular formulation variant of the well known complex impedance formalism typically used for the electric characterization of dielectric, ferroelectric, and piezoelectric materials. It has been successfully exploited as a versatile tool for characterization of the magnetization dynamics in amorphous ribbons and wires by means of simple experiments involving coils for sample holding and impedance analyzer equipment. This technique affords the resolution of the magnetization processes in soft magnetic materials, in terms of reversible deformation of pinned domain walls, domain wall displacements and spin rotation, for which characteristic parameters such as the alloy initial permeability and the relaxation frequencies, indicating the dispersion of each process, can be defined. Additionally, these parameters can be correlated with chemical composition variation, size effects and induced anisotropies, leading to a more physical insight for the understanding of the frequency dependent magnetic response of amorphous alloys, which is of prime interest for the development of novel applications in the field of telecommunication and sensing technologies. In this work, a brief overview, together with recent progress on the magnetization dynamics of amorphous ribbons, wires, microwires and biphase wires, is presented and discussed for the intermediate frequency interval between $10 \mathrm{~Hz}$ and $13 \mathrm{MHz}$.
\end{abstract}

Keywords: amorphous alloys; magnetization dynamics; magnteoinductance; amorphous ribbons; amorphous wires 


\section{Introduction}

Amorphous alloys obtained from rapid solidification techniques as thin ribbons (with characteristic thickness below 30 microns and variable lengths) and fine wires (with typical diameters lower than 120 microns and variable lengths) have been the subject of research since the 1980s due to their ultrasoft magnetic character which has allowed interesting technological applications in devices for conversion of electromagnetic energy into mechanic energy and for signal processing; power electronics, electric power conditioning, magnetic sensors, telecommunication, automotive magnetics; and electronic article surveillance [1,2]. Amorphous ribbons can be cast by means of chill block melt spinning method which involves the continuous ejection of a molten alloy onto the edge surface of a wide rotating copper disk (with typical angular speeds within the range 2,000-3,000 rpm) which allows solidification rates between $10^{5}-10^{7} \mathrm{~K} / \mathrm{s}$ [3]. On the other hand, for the formation of wires with diameters within the range 80-120 microns, a variant of melt spinning process known as "rotating water bath melt spinning" entails the streaming of a fine jet of molten alloy into a flow of cold water (moving in a rapidly rotating drum at almost the same velocity as the jet) such that the alloy rapidly solidifies and vitrifies [4]. In addition, amorphous glass coated microwires, with usual diameters from 5 to 40 microns for the metallic nucleus and between 5-25 microns for the surrounding glass layer, can be produced by a modified Taylor-Ulitovsky technique in which a metal drop melted by high frequency induction is placed inside a glass tube. The temperature achieved by the melting assembly is high enough to allow the softening of the glass tube and its further drawing to a thin capillary which in turn is wound onto a receiving bobbin. The fast moving capillary entrapping the melted alloy passes through a cooling water jet for its rapid solidification [5].

Usually, within the study of soft magnetic materials, the characterization of the magnetic permeability $\mu$ as a function of frequency is a figure of merit because of the possibility for developing high frequency applications for telecommunications, wireless systems and radar detection, among others. In this context, a powerful characterization technique is the so called impedance spectroscopy (ImS) which has been widely used to investigate electric polarization phenomena in a variety of dielectric, ferroelectric, and piezoelectric materials [6]. However, it can be shown that by a simple change in experimental conditions (essentially coils instead of electrodes for applying external ac magnetic fields $h_{\mathrm{ac}}$ of variable intensity), and by a convenient transformation of complex formalisms (inductances and magnetic permeabilities instead of capacitances and permittivities), it is possible to build a methodology which can be established as inductance spectroscopy (InS) [7]. As in ImS, for materials characterization by $\mathrm{InS}$, it is feasible to resolve the dominant magnetization processes by measuring in a wide frequency range. At low frequencies, all the magnetization processes (namely reversible domain wall bulging, domain wall displacement, spin rotation and spin precession) contribute to the total value permeability (and thus, of magnetization). However, they possess different dynamics in the sense that each magnetization process is characterized by a time-constant. As the frequency of $h_{\mathrm{ac}}$ increases, the magnetization processes with slow dynamics (i.e., large time-constants) become unable to follow the excitation field exhibiting a dispersion and subsequently, a decrease in the total value of permeability. The permeability value in each frequency range, the frequency at which the process ceases to follow the field, and the dispersion (the behavior to change from one magnetization process to another one) are significant aspects to understand the frequency response of 
the material. A wide variety of magnetic materials have been investigated by InS, such as ferrites [8], amorphous and nanocrystallized ribbons [9], amorphous wires [10] and glass-coated microwires [11].

The InS formalism involves the calculation of the complex inductance $\mathbf{L}=L_{\mathrm{re}}+\mathrm{i} L_{\mathrm{im}}(\mathrm{i}=\sqrt{ }-1)$ from complex impedance measurements $\mathbf{Z}=Z_{\mathrm{re}}+\mathrm{i} Z_{\mathrm{im}}$ via the following transformation

$$
\mathbf{L}=-\mathrm{i} \mathbf{Z} / \omega
$$

where $\omega$ is the angular frequency of $h_{a c}$. Complementarily, the complex permeability formalism $\mu=\mu_{\mathrm{re}}+\mathrm{i} \mu_{\mathrm{im}}$ can be formulated according to

$$
\boldsymbol{\mu}=G \mathbf{L}
$$

where $G$ is an appropriate geometrical factor. For instance, $G \sim 10^{8}$ (Henry) $^{-1}$ for wires and microwires [12]. Within both of these formalisms, the real component $L_{\mathrm{re}}$ (or $\mu_{\mathrm{re}}$ ) corresponds to the material magnetic permeability, whilst $L_{\mathrm{im}}\left(\right.$ or $\left.\mu_{\mathrm{im}}\right)$ is associated with the dissipative process. The spectroscopic plots $L_{\mathrm{re}}(\omega)$ and $L_{\mathrm{im}}(\omega)$ (or alternatively $\mu_{\mathrm{re}}(\omega)$ and $\mu_{\mathrm{im}}(\omega)$ ) obtained from Equations (1) and (2) have led to the resolution as a function of frequency of the following magnetization mechanisms: Reversible domain wall bulging, unpinning-displacement of domain walls (i.e., hysteresis) and spin rotation. At frequencies low enough, all these magnetization processes contribute to the material's response; in particular, domain wall movements and spin rotation

An additional aspect to bear in mind for the dynamic characterization of the magnetization in amorphous ribbons and wires, is the particular magnetic domain formation features of these types of alloys. Such magnetic domain distribution strongly depends on the magnetoelastic coupling between the residual stresses frozen during the rapid solidification process and the sign and magnitude of the alloy magnetostriction $\lambda_{\mathrm{s}}[13,14]$. For the case of ribbons, longitudinal internal stresses (i.e., along the length direction of the sample) develop as a consequence of the gradient of temperature between the wheel contact-side of the ribbon and it's free-side [3] leading to in-plane-easy axis and hence, longitudinal magnetic domains are preferentially formed for Fe-based ribbons with high $\lambda_{\mathrm{s}}$ constants ( $\left.\lambda_{\mathrm{s}} \sim 10-30 \mathrm{ppm}\right)$. In contrast, negative sign of $\lambda_{\mathrm{s}}$ favors the appearance of transverse magnetic domains (i.e., perpendicularly oriented respect to the ribbon main axis). Nevertheless, for both cases, the residual stresses follow a rather complex distribution, including in-plane tensile and compressive stresses, but also planar compressive stress inducing perpendicular easy axis (relative to the ribbon surface). These internal stresses can vary not only laterally, but also in depth, leading to the formation of rather complicated magnetic domain configurations including: Wide curved domains with $180^{\circ}$ walls, narrow fingerprints, maze and dense stripe domains $[15,16]$. On the other hand, for amorphous wires the magnetoelastic properties are also determined by the complex quenched-in stresses configuration arising from the casting process, especially from the strong thermal gradient between the inner and the outer regions of the wire, together with the sign of $\lambda_{s}$. Additionally, for amorphous glass covered microwires, there are two kinds of stress sources (a) axial stress due to the cooling process and (b) radial stress caused by the difference in thermal expansion coefficients between the metallic nucleus and the glass coating [17,18]. These internal stress variations follow a rather complex distribution with axial, circular and radial components changing rapidly from positive (at the axial zone) to negative (at the surrounding shell) including maximum values at half the radius, as well as on the wire edges, and even zero stress value is expected at the precise microwire centre $[19,20]$. In any 
case, the sign of $\lambda_{\mathrm{s}}$ determines again the peculiar magnetic domain configuration for these kinds of amorphous alloys: An inner zone with magnetic axial orientation surrounded by a sheath of perpendicular magnetization with either radial (a characteristic of Fe-based wires with large positive $\lambda_{\mathrm{s}}$ ) or circular orientation (typical of Co- and CoFe-based wires with negative $\lambda_{\mathrm{s}}$ ) $[18,21]$. In this work, recent progress on the magnetization dynamics, in terms of the resolution of magnetization processes as a function of frequency, for amorphous ribbons and wires, is presented and discussed

\section{Results and Discussion}

\subsection{Amorphous Ribbons}

The spectroscopy behavior for real and imaginary components of complex permeability for amorphous $\mathrm{Fe}_{80} \mathrm{~B}_{10} \mathrm{Si}_{10}$ ribbons are shown in Figure 1. These curves afford the resolution of the active magnetization mechanisms across the frequency range for a given $h_{\mathrm{ac}}$ intensity [22,23]. For instance, the $\mu_{\mathrm{re}}(f)$ plot for an $h_{\mathrm{ac}}=0.42 \mathrm{~A} / \mathrm{m}$ shows an initial plateau-like behavior for increasing frequency $f$ values up to $6 \times 10^{4} \mathrm{~Hz}$. This tendency of $\mu_{\mathrm{re}}$ can be attributed to the reversible bulging of the magnetic domain walls (DWs) pinned at the ribbon surface's defects (such as voids, surface irregularities and the very surface itself). Hence, $\mu_{\mathrm{re}}$ at this part of the frequency is then associated with the initial permeability $\mu_{\text {ini }}$ of the material [22,24] Further, increase in $f$ causes a significant reduction in $\mu_{\mathrm{re}}(f)$, which can be ascribed to a relaxation-type dispersion of the reversible bulging mechanism for which the DWs are no longer able to follow the ac magnetic variations. Beyond a threshold frequency $f_{\mathrm{x}}$ (or relaxation frequency) $\mu_{\text {re }}$ becomes very small, reflecting the contribution of the spin rotation as the only magnetization process active for $f>f_{\mathrm{x}}[22,24]$. Complementarily, the imaginary $\mu_{\mathrm{im}}$ component is attributed to the alloy magnetic losses (hysteresis, eddy current, power losses) $[25,26]$. The maximum in $\mu_{\mathrm{im}}$ corresponds to $f_{\mathrm{x}}$.

Figure 1. Spectroscopic plots of the complex permeability components for amorphous $\mathrm{Fe}_{80} \mathrm{~B}_{10} \mathrm{Si}_{10}$ ribbon at $h_{\mathrm{ac}}=0.42$.

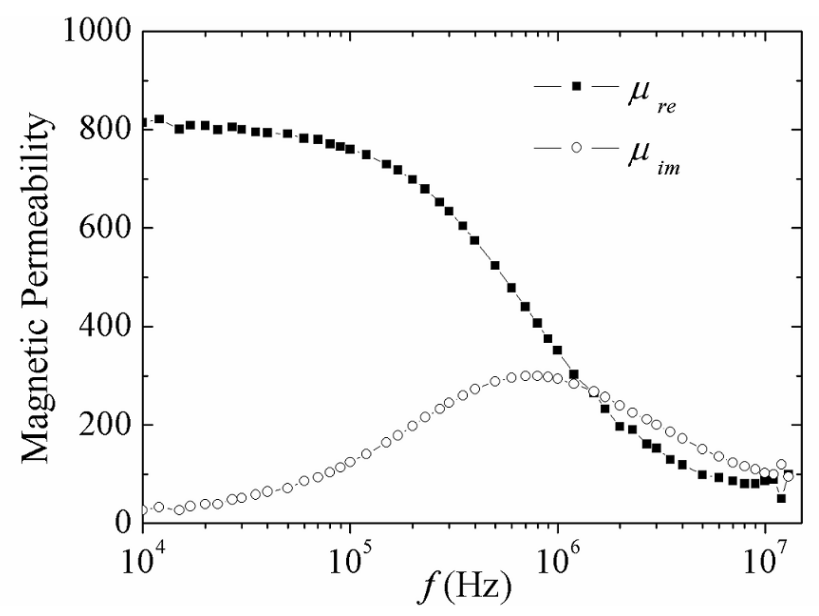

On the other hand, for a higher $h_{\mathrm{ac}}$ intensity of $1.69 \mathrm{~A} / \mathrm{m}$, it is possible to observe two sections of the $\mu_{\mathrm{re}}(f)$ plot as is shown in Figure 2 for the same FeBSi ribbons: The reversible bulging process, together with its corresponding relaxation for $f>1 \times 10^{5} \mathrm{~Hz}$, and a significant increase in $\mu_{\mathrm{re}}$ for the 
frequency interval $3 \times 10^{3} \mathrm{~Hz}<f<1 \times 10^{5} \mathrm{~Hz}$, with a precedent constant tendency for $f<3 \times 10^{3} \mathrm{~Hz}$. This increment in $\mu_{\mathrm{re}}$ can be interpreted in terms of a magnetization mechanism involving the irreversible displacement of DWs caused by the higher $h_{\text {ac }}$ intensity [22-24]. The DWs displacement leads to the hysteresis process within the material, which also presents a relaxation-type dispersion (i.e., a change of process) for the aforementioned frequency interval $\left(3 \times 10^{3} \mathrm{~Hz}<f<1 \times 10^{5} \mathrm{~Hz}\right)$, for which a progressive decrease in $\mu_{\mathrm{re}}$ is manifested for increasing $f$ up to a threshold frequency known as "hysteresis relaxation frequency" $f_{\mathrm{x}}^{\mathrm{h}}$. Beyond this $f_{\mathrm{x}}^{\mathrm{h}}$, the irreversible displacement of DWs becomes unable to follow the exciting $h_{\mathrm{ac}}$ field, allowing the reversible bulging to be the active magnetization process because of its shorter time constant. Complementarily, the imaginary component $\mu_{\mathrm{im}}(f)$ exhibits a behavior consistent with the sequence of magnetization mechanisms described already, showing one maximum for each process, i.e., irreversible displacement of DWs at $f_{\mathrm{x}}^{\mathrm{h}}$ and reversible bulging of DWs at $f_{\mathrm{x}}$, respectively. For this case $f_{\mathrm{x}}{ }^{\mathrm{h}}=8 \times 10^{3} \mathrm{~Hz}$ and $f_{\mathrm{x}}=8 \times 10^{5} \mathrm{~Hz}$. The marked order of magnitude between both threshold frequencies $\left(f_{\mathrm{x}}^{\mathrm{h}}<<f_{\mathrm{x}}\right)$ reflects the significant difference between the time constant for each magnetization mechanism (much larger for the hysteresis process compared with the reversible bulging of DWs). In addition, the magnetic losses (proportional to the area under the $\mu_{\mathrm{im}}$ curve) are also comparatively larger for the irreversible displacement of DWs than for the reversible bulging, due to the higher energy cost for the hysteresis process relative to the reversible deformation of the DWs.

Figure 2. Spectroscopic plots of the complex permeability components for amorphous $\mathrm{Fe}_{80} \mathrm{~B}_{10} \mathrm{Si}_{10}$ ribbon at $h_{\mathrm{ac}}=1.69 \mathrm{~A} / \mathrm{m}$.

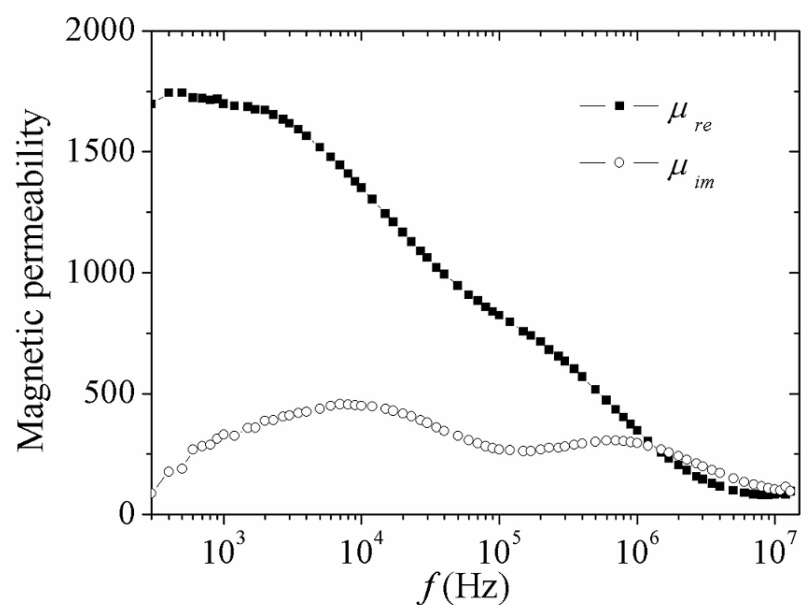

The magnetic permeability and thus, the magnetization processes, are highly sensitive to the chemical composition of the alloy ribbons because of the possible effect on the short-range atomic ordering introduced by variable atom species, which may affect the exchange interaction between magnetic moments as well as the formation of spin-up/spin down bands [27-29]. In Figure 3, the real permeability spectroscopic plots (Figure 3a) for melt spun $\mathrm{Fe}_{80} \mathrm{~B}_{10} \mathrm{Si}_{10-\mathrm{x}} \mathrm{Ge}_{\mathrm{x}}(\mathrm{x}=0.0-10.0)$ ribbons shows an initial behavior $(\mathrm{x}=0.0)$ including irreversible DWs displacement for $f<1 \times 10^{5} \mathrm{~Hz}$ and its further reversible bulging response for higher frequencies. Nevertheless, for increasing $\mathrm{Ge}$ concentration $(\mathrm{x} \geq 2.5)$ the low time constant processes (i.e., hysteresis) disappears, leaving the reversible bulging of DWs as the only active mechanism, for which a progressive deleterious tendency 
is observed with increasing Ge content. Complementarily, the imaginary component $\mu_{\mathrm{im}}(f)$ (Figure $3 \mathrm{~b}$ ) also exhibits a transition from a double-peak behavior corresponding to two magnetization processes, to a single maximum regime associated to the magnetization mechanism with higher time constant. Additionally, it is possible to observe that the relaxation frequency $f_{\mathrm{x}}$ increases in a monotonous manner with increasing $\mathrm{x}$, as indicated by the maxima in $\mu_{\mathrm{im}}(f)$ plots moving to higher frequency values. These results point to a significant compositional influence on the time constant response of amorphous alloys via the effective magnetic anisotropy, which has been observed also in amorphous wires [30] and soft ferrites [31].

Figure 3. Spectroscopic plots of the complex permeability components for the amorphous ribbon $\mathrm{Fe}_{80} \mathrm{~B}_{10} \mathrm{Si}_{10-\mathrm{x}} \mathrm{Ge}_{\mathrm{x}}$ series at $h_{\mathrm{ac}}=1.69 \mathrm{~A} / \mathrm{m}$.

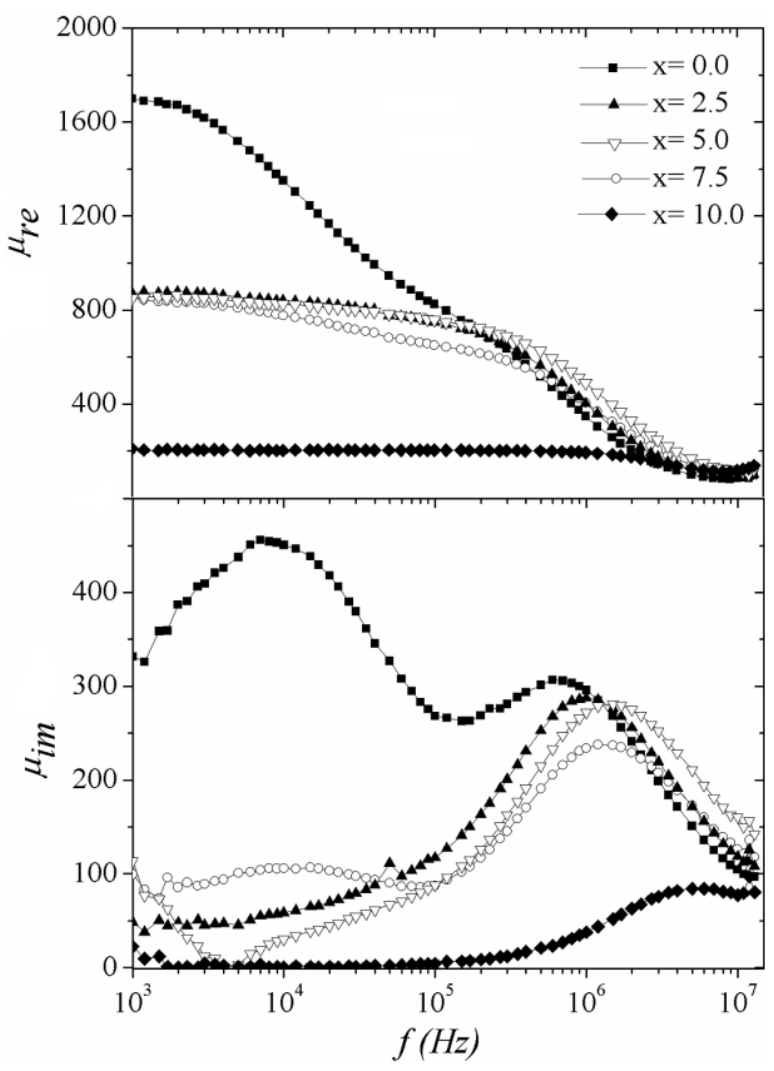

As was mentioned in the "Introduction" section, the magnetoelastic coupling between the residual stresses and the alloy magnetostriction $\lambda_{\text {s }}$ plays a major role in determining the magnetic domain configuration of ribbons and wires and, hence, of their magnetic properties. In order to monitor the magnetoelastic coupling for the ribbon series $\mathrm{Fe}_{52-\mathrm{x}} \mathrm{Co}_{10+\mathrm{x}} \mathrm{Nb}_{8} \mathrm{~B}_{30}(\mathrm{x}=0,12,24,36), \mu_{\text {re }}$ plots as a function of an applied magnetic dc field $\mathrm{H}_{\mathrm{dc}}$ and under the effect of variable longitudinal applied tension stress $\sigma$ at a constant frequency $f=13 \mathrm{MHz}$ were determined for each composition. Figure 4 illustrates $\mu_{\mathrm{re}}\left(\mathrm{H}_{\mathrm{dc}}\right)-\sigma$ curves for the $\mathrm{Fe}_{52} \mathrm{Co}_{10} \mathrm{Nb}_{8} \mathrm{~B}_{30}$ alloy $(\mathrm{x}=0)$, for which at $\sigma=0$, $\mu_{\mathrm{re}}$ initially increases for $\mathrm{H}_{\mathrm{dc}}$ values between 0 and $800 \mathrm{~A} / \mathrm{m}$, followed by a monotonous decrease for $\mathrm{H}_{\mathrm{dc}}>800 \mathrm{~A} / \mathrm{m}$. This behavior is symmetrical for negative $\mathrm{H}_{\mathrm{dc}}$. For increasing $\sigma$, this original two-peak behavior progressively evolves to a reduced single-maximum $\mu_{\mathrm{re}}\left(\mathrm{H}_{\mathrm{dc}}\right)$ picture. Same tendencies were 
observed for the remaining alloy samples, except for the $\mathrm{x}=36$ alloy, which showed negligible magnetic permeability values as a consequence of its vanishing $\lambda_{\mathrm{s}}$ constant.

Figure 4. $\mu_{\mathrm{re}}\left(\mathrm{H}_{\mathrm{dc}}\right)$ curves under the effect of variable tension stress $\sigma$ for the $\mathrm{Fe}_{52} \mathrm{Co}_{10} \mathrm{Nb}_{8} \mathrm{~B}_{30}$ ribbon.

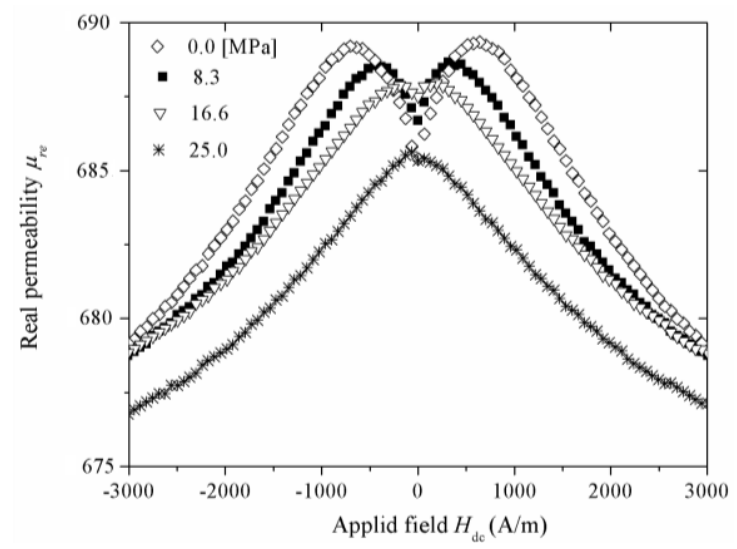

The physical meaning of these $\mu_{\mathrm{re}}\left(\mathrm{H}_{\mathrm{dc}}\right)-\sigma$ plots can be explained as follows: At the frequency of measurement $\left(f=13 \mathrm{MHz} \gg f_{\mathrm{x}}\right.$ ), the spin rotation remains as the only active magnetization mechanism and thus, the real component of permeability corresponds to the rotational permeability $\mu_{\mathrm{re}}{ }^{\text {rot }}$. For the stress-free curve, the initial increase of $\mu_{\mathrm{re}}{ }^{\text {rot }}$ up to $\mathrm{H}_{\mathrm{dc}}=800 \mathrm{~A} / \mathrm{m}$ can be ascribed to an additional component (axially oriented) of $\mu_{\mathrm{re}}^{\text {rot }}$ exerted by $\mathrm{H}_{\mathrm{dc}}$. The maximum in $\mu_{\mathrm{re}}{ }^{\text {rot }}$ is a consequence of a counterbalancing effect in the rotational magnetization process between the axial $\mathrm{H}_{\mathrm{dc}}$ and the anisotropy field $H_{\mathrm{k}}$ in the transverse direction induced during the ribbon fabrication. Thus, the maximum in $\mu_{\mathrm{re}}{ }^{\text {rot }}\left(\mathrm{H}_{\mathrm{dc}}\right)$ corresponds to $\mathrm{H}_{\mathrm{k}}[32,33]$. For $\mathrm{H}_{\mathrm{dc}}$ beyond $800 \mathrm{~A} / \mathrm{m}$, the rotational permeability decreases since the magnetic spins are mostly axially oriented after having overcome the original anisotropy. On the other hand, for the $\mu_{\mathrm{re}}{ }^{\text {rot }}\left(\mathrm{H}_{\mathrm{dc}}\right)$ curves with increasing $\sigma$ (up to 16.6 MPa), a shifting of $H_{\mathrm{k}}$ towards lower values is observed as a consequence of a progressively higher induced axial anisotropy, allowed by the increment of $\sigma$, which counterbalance the original transverse anisotropy and thus, the as-cast transverse magnetoelastic coupling. For $\sigma=25.0 \mathrm{MPa}$, the alloy transverse anisotropy is averaged out, which results in a single-peak regime with reduced $\mu_{\mathrm{re}}{ }^{\text {rot }}$ as a consequence of the predominant axial anisotropy induced by $\sigma$. This behavior is indicative of a positive $\lambda_{\mathrm{s}}$. The dependence of $H_{\mathrm{k}}$ on $\sigma$ for alloy ribbons with $\mathrm{x} \leq 36$ is displayed in Figure 5, for which the following linear trends are manifested (after appropriate linear fittings): $\mathrm{H}_{\mathrm{k}}=576[\mathrm{~A} / \mathrm{m}]-27.3 \times 10^{-6}[\mathrm{~A} /(\mathrm{mPa})]$ $\sigma \mathrm{x}=0 ; \mathrm{H}_{\mathrm{k}}=628[\mathrm{~A} / \mathrm{m}]-10.3 \times 10^{-6}[\mathrm{~A} /(\mathrm{mPa})] \sigma, \mathrm{x}=12 ;$ and $\mathrm{H}_{\mathrm{k}}=387[\mathrm{~A} / \mathrm{m}]-17.7 \times 10^{-6}[\mathrm{~A} /(\mathrm{mPa})]$ $\sigma, x=24$. 
Figure 5. Anisotropy field $H_{\mathrm{k}}$ as a function of applied stress $\sigma$ for the $\mathrm{Fe}_{52-\mathrm{x}} \mathrm{Co}_{10+\mathrm{x}} \mathrm{Nb}_{8} \mathrm{~B}_{30}$ alloy series. The straight lines correspond to linear fittings with at least $\mathrm{R}^{2}=0.99$.

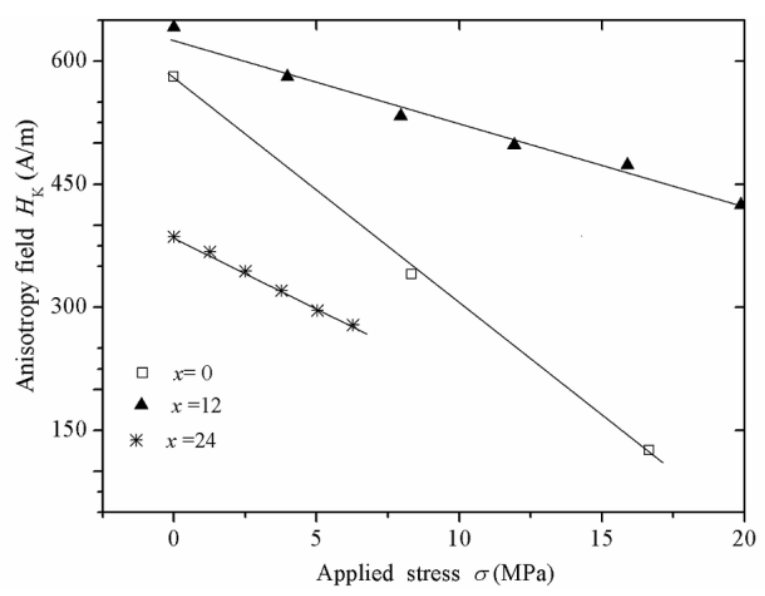

From the functional relations stated above, it is possible to estimate the $\lambda_{\mathrm{s}}$ constant for each alloy according to the following relationship [33,34]:

$$
\lambda_{s}=-\left(\frac{\mu_{o} M_{s}}{3}\right)\left(\frac{\Delta H_{z}}{\Delta \sigma}\right)
$$

where $\mu_{0} M_{\mathrm{s}}$ is the saturation magnetization and $\Delta \mathrm{H}_{\mathrm{z}}$ corresponds to the variations of the transverse field $\mathrm{H}_{\mathrm{z}}$ necessary to compensate the magnetoelastic contribution induced by $\sigma$. This equation is proposed for positive and negative $\lambda_{\mathrm{s}}$ values $[33,34]$. For present measurements, the compensating $\Delta \mathrm{H}_{\mathrm{z}}$ field corresponds to $H_{k}$ straightforward. Therefore, Equation (3) becomes:

$$
\lambda_{s}=-\left(\frac{\mu_{o} M_{s}}{3}\right)\left(\frac{H_{k}}{\Delta \sigma}\right)
$$

Taking $\mu_{\mathrm{o}}=12.56 \times 10^{-7} \mathrm{H} / \mathrm{m}$ and the following $\mu_{0} M_{\mathrm{s}}$ values determined from $M-H$ measurements in a VSM system: $1.24 \mathrm{~T}(\mathrm{x}=0), 1.09 \mathrm{~T}(\mathrm{x}=12), 0.86 \mathrm{~T}(\mathrm{x}=24)$, in addition to $H_{\mathrm{k}} / \Delta \sigma$ as the slopes of the $\mathrm{H}_{\mathrm{k}}(\sigma)$ plots (Figure 5), we found a decreasing $\lambda_{\mathrm{s}}$ tendency with increasing Co concentration as follows: $\lambda_{\mathrm{s}}=10.7 \mathrm{ppm}(\mathrm{x}=0), \lambda_{\mathrm{s}}=3.6 \mathrm{ppm}(\mathrm{x}=12)$ and $\lambda_{\mathrm{s}}=3.0 \mathrm{ppm}(\mathrm{x}=24)$.

\subsection{Amorphous Wires}

An alternative representation of the spectroscopic $\mu_{\mathrm{re}}(f), \mu_{\mathrm{im}}(f)$ plots (or alternatively $L_{\mathrm{re}}(f), L_{\mathrm{im}}(f)$ plots) is the so-called Cole-Cole diagram in which both components are plotted simultaneously in the complex plane, with the frequency $f$ being now an implicit variable (the corresponding $f$ for each data point increases from right to left in the plot) as is shown in Figure 6 for an amorphous wire of composition $\left(\mathrm{Co}_{94} \mathrm{Fe}_{6}\right)_{72.5} \mathrm{~B}_{15} \mathrm{Si}_{12.5}(120 \mu \mathrm{m}$ diameter). For this representation, the formation of well defined semicircles is associated with a magnetization process [23]. In the case of Figure 6, a single semicircle, somehow distorted, reveals the reversible bulging of DWs within the wire as the active mechanism for the frequency range of measurements $(10 \mathrm{~Hz}-13 \mathrm{MHz})$. The dispersion of the process for the Cole-Cole representation occurs at the middle point onto the semicircle. For this case, $f_{\mathrm{x}}=88.5 \mathrm{kHz}$. The distortion of the semicircle reflects a distribution of time constants within the wire, i.e., variations 
of the DWs pinning distance. For a homogenous DWs pinning distance, a perfect circular curvature is expected to occur in the Cole-Cole plane [23].

Figure 6. Cole-Cole representation for the complex inductance formalism of $\left(\mathrm{Co}_{0.94} \mathrm{Fe}_{0.6}\right)_{72.5} \mathrm{~B}_{15} \mathrm{Si}_{12.5}$ amorphous wire.

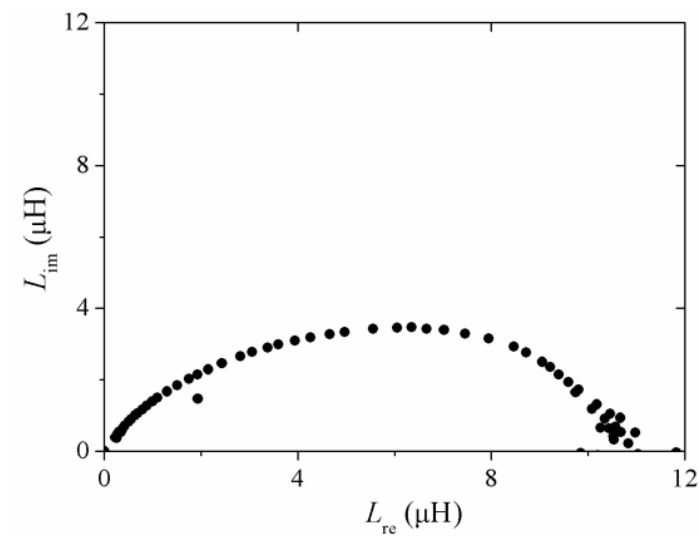

A more physical insight into the magnetization processes involved in $\mathrm{InS}$ measurements can be attained by means of direct calculation of the circular magnetization $M_{\phi}$ of amorphous wires, which is afforded by the circular magnetic domains characteristic of these alloys. The $\mathbf{M}_{\phi}$ determination is described as follows:

The magnetic permeability calculation of Equation (2) corresponds to circular permeability $\mu_{\phi}$ in amorphous wires, since the applied magnetic field $h_{\mathrm{ac}}$ is also of circumferential character as described in the "Experimental Techniques" section. Once $\mu_{\phi}$ is known, the wire's circular induction $\mathrm{B}_{\phi}$ can be worked out from the permeability definition:

$$
\mu_{\phi}=\mathrm{B}_{\phi} / h_{a c}
$$

and from the general equation

$$
\mathrm{B}_{\phi}=\mu_{\mathrm{o}}\left(\mathrm{M}_{\phi}+h_{a c}\right)
$$

with $\mu_{\mathrm{o}}$ the vacuum's permeability.

The calculation of the circular magnetization $\mu_{0} \mathbf{M}_{\phi}$ is straightforward according to:

$$
\mu_{\mathrm{o}} \mathrm{M}_{\phi}=h_{a c}\left(k \mathrm{~L}_{\mathrm{r}}-\mu_{\mathrm{o}}\right)
$$

Therefore, circular magnetization plots $\mu_{0} \mathbf{M}_{\phi}\left(h_{a c}\right)$ can be calculated for various $h_{a c}$ amplitudes at fixed frequencies $f$ of $h_{a c}$, as is shown in Figure 7, for an amorphous $\left(\mathrm{Co}_{94} \mathrm{Fe}_{6}\right)_{72.5} \mathrm{~B}_{15} \mathrm{Si}_{12.5}$ wire with vanishing $\lambda_{s}$. At low frequency (i.e., $f<<f_{x}$ ), a typical domain wall magnetization process is observed at the linear initial $\mathrm{M}_{\phi}-h_{\mathrm{ac}}$ behavior, corresponding to reversible pinned domain wall bulging, followed by a sudden increase in $\mu_{o} \mathrm{M}_{\phi}$ at a particular $h_{a c}$ field value known as the propagation field $h_{p}$ for which the irreversible wall displacement begins. Further increase in $h_{a c}$ amplitude should exhibit a $\mu_{0} \mathbf{M}_{\phi}$ trend to saturation, where the spin rotation will complete the magnetic moment alignment towards $h_{a c}$ orientation. On the other hand, for $f \gg f_{x}$, the linear character of the magnetization curve across the $h_{a c}$ amplitude variation, clearly reflects the spin rotation as the only magnetization mechanism remaining active at such a frequency. 
Figure 7. Circular magnetization plots $\mathrm{M}_{\phi}\left(h_{a c}\right)$ for $\left(\mathrm{Co}_{0.94} \mathrm{Fe}_{0.6}\right)_{72.5} \mathrm{~B}_{15} \mathrm{Si}_{12.5}$ amorphous wire.

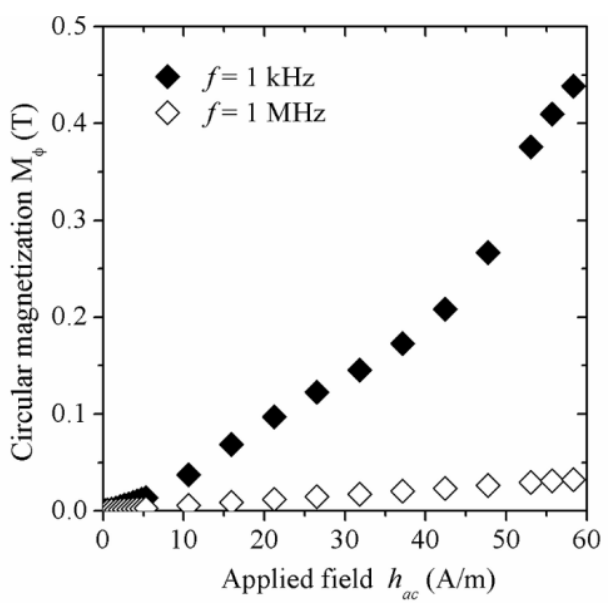

An interesting set of measurements aiming to determine the effect of torsion strain $\zeta$ on the wire's circular magnetization at low frequency $(f=1 \mathrm{kHz})$ is shown in Figure 8. For positive $\zeta$ (clockwise rotation, Figure $8 \mathrm{a}), \mu_{0} \mathrm{M}_{\phi}$ versus $h_{a c}$, increases with the torsion angle up to a maximum of $120^{\circ}$. Further increase in $\zeta$ results in decreasing $\mu_{0} \mathrm{M}_{\phi}$. Counterclockwise rotations ( $\zeta$, Figure $8 \mathrm{~b}$ ), on the other hand, resulted in decreasing $\mu_{0} \mathbf{M}_{\phi}$ as $\zeta$ increases.

Figure 8. $\mathrm{M}_{\phi}\left(h_{a c}\right)$ plots for $\left(\mathrm{Co}_{0.94} \mathrm{Fe}_{0.6}\right)_{72.5} \mathrm{~B}_{15} \mathrm{Si}_{12.5}$ amorphous wire at variable positive and negative torsion angles.

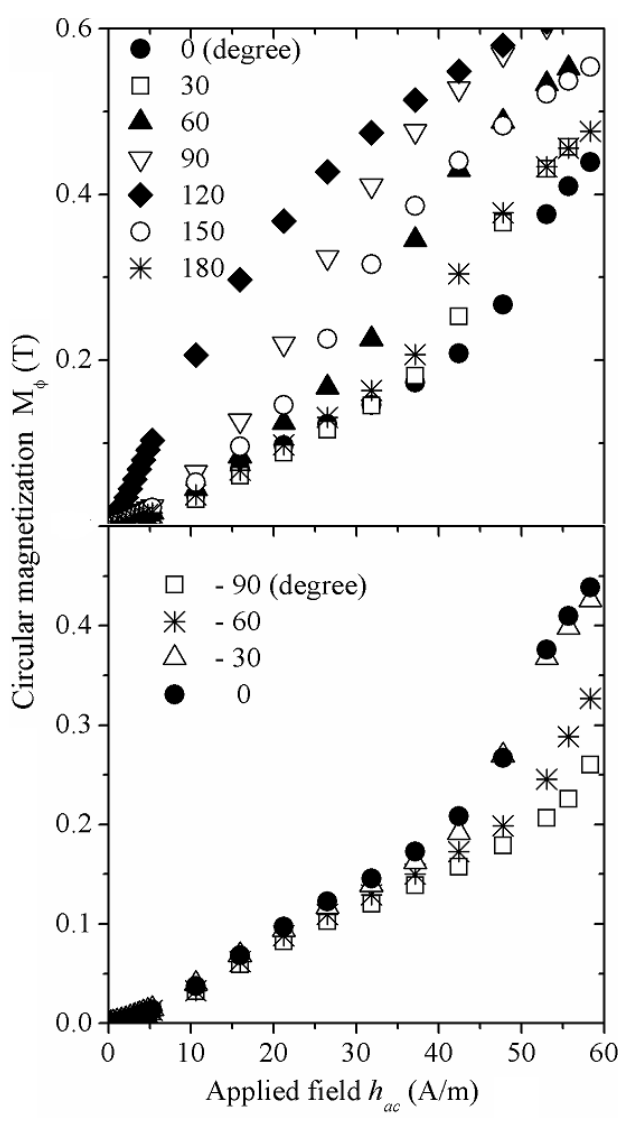


In addition, $h_{p}$ (calculated at the inflexion point of each $\mu_{o} \mathbf{M}_{\phi}\left(h_{a c}\right)$ plot), exhibits a sharp minimum (Figure 9) at the same torsion angle. This particular $\mu_{0} \mathrm{M}_{\phi}, h_{p}$ behavior with varying torsion angles can be interpreted in terms of an intrinsic helical anisotropy induced during the fabrication process, which results in internal stresses $\tau$, of about $60 \mathrm{MPa}$, according to theoretical predictions based on cylindrical layer solidification models $[35,36]$. Then, as $+\zeta$ increases, a counterbalance effect of this $+\zeta$ against the internal stress is evident, since the wire becomes magnetically softer, which affords higher $\mu_{0} \mathrm{M}_{\phi}$ values (due to higher $\mu_{\phi}$ ) together with lower $h_{p}$ values, as a consequence of an enhancement of the unpinning-displacement domain wall process. In contrast, for negative torsion angles, progressive increase in induced anisotropy due to $-\zeta$ results in decreasing $\mu_{\phi}$ and, hence, in lower $\mu_{0} M_{\phi}$ values, is in agreement with recent reports [37]. The corresponding applied torsion stress $\tau_{\text {appl }}$, for a torsion angle of $120^{\circ}$, can be calculated as follows:

$$
\tau=\mathrm{K} \zeta \mathrm{r}
$$

where $\mathrm{K}$ is the wire's shear modulus, $\zeta=$ (torsion angle)/(wire length) is the torsion strain and $\mathrm{r}$ is the wire radius. Using $\mathrm{K}=60 \mathrm{GPa}$ [38], we have $\tau_{\text {appl }}=83 \mathrm{MPa}$, which is in good agreement with the expected internal $\tau$ induced during the wire fabrication (between 50 and $100 \mathrm{MPa}$ [39]). Similar $\tau_{\text {appl }}$ for enhanced $\mu_{\phi}$ at various $\zeta$ values has also been found in Fe-based wires [40].

Figure 9. Propagation field $h_{p}$ as a function of the torsion angle for amorphous $\left(\mathrm{Co}_{0.94} \mathrm{Fe}_{0.6}\right)_{72.5} \mathrm{~B}_{15} \mathrm{Si}_{12.5}$ wire.

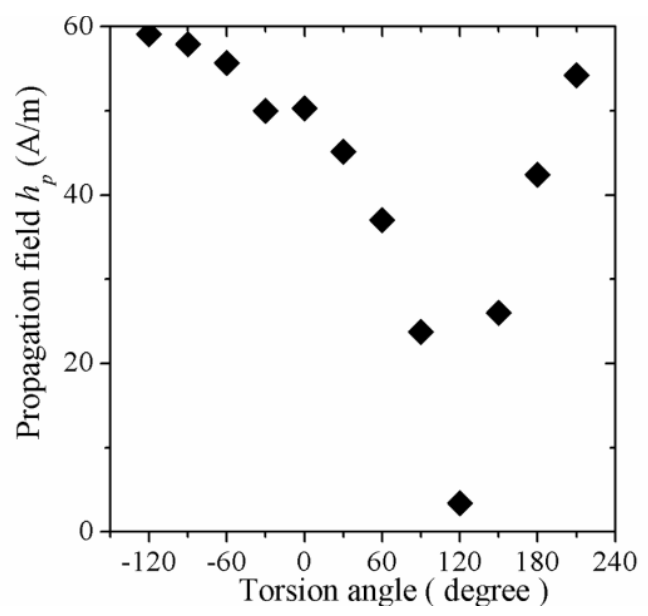

\subsection{Amorphous Glass Coated Microwires}

Spectroscopic plots of $\mu_{\text {re }}(f)$ for amorphous $\mathrm{Co}_{69.7} \mathrm{Fe}_{3.8} \mathrm{~B}_{13} \mathrm{Si}_{11} \mathrm{Mo}_{1.5} \mathrm{Ni}_{1}$ glass coated microwires (24 $\mu \mathrm{m}$ diameter, $10 \mu \mathrm{m}$ glass coating) at selected amplitudes of $h_{\mathrm{ac}}$, are shown in Figure 10. At low fields $\left(h_{a c}<127 \mathrm{~A} / \mathrm{m}\right)$ and low frequencies $(<1 \mathrm{MHz}), \mu_{\mathrm{re}}$ exhibits the same constant trend even for different $h_{a c}$ amplitudes, followed by its corresponding dispersion. The relaxation frequency $f_{\mathrm{x}}$ again represents the frequency for which the initial magnetization mechanism is no longer active. This $f_{x}$ can be determined at the corresponding $\mu_{\mathrm{im}}$ maximum (Figure 11). For the present case, $f_{x}=2.5 \mathrm{MHz}$. This $f_{x}$ value is much higher than the typical $f_{\mathrm{x}}$ values of amorphous wires with larger diameters values (of about $100-140 \mu \mathrm{m}$ ) with characteristic $f_{\mathrm{x}} \sim 90 \mathrm{kHz}$. The substantial increase of $f_{\mathrm{x}}$ for amorphous glass coated microwires is a consequence of their reduced diameter, since the DWs lengths are smaller than 
those in larger amorphous wires, and therefore, the expected relaxation occurs at higher frequencies. For $h_{\mathrm{ac}}>127 \mathrm{~A} / \mathrm{m}, \mu_{\mathrm{re}}$ is no longer independent of $h_{\mathrm{ac}}$ amplitude (at $f<f_{\mathrm{x}}$ ). For $f>f_{\mathrm{x}}$, all the plots converge into the low field plot exhibiting the same relaxation dispersion. Assuming a domain structure consisting of circumferential walls for these Co-based microwires, the magnetization process for $h_{\mathrm{ac}}<127 \mathrm{~A} / \mathrm{m}$ is associated with reversible bulging of domain walls since these DWs are considered to be pinned to surface defects.

Figure 10. Spectroscopic plots $\mu_{\mathrm{re}}(f)$ for glass coated $\mathrm{Co}_{69.7} \mathrm{Fe}_{3.8} \mathrm{~B}_{13} \mathrm{Si}_{11} \mathrm{Mo}_{1.5} \mathrm{Ni}_{1}$ microwire with $h_{\mathrm{ac}}$ amplitude as a parameter.

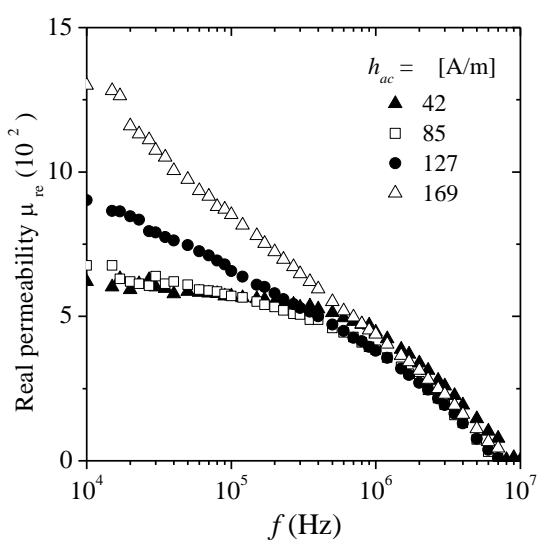

The frequency response of $\mu_{\mathrm{im}}$ for various $h_{\mathrm{ac}}$ amplitudes is shown in Figure 11 . For $h_{\mathrm{ac}}<127 \mathrm{~A} / \mathrm{m}$, a single peak is observed, which is ascribed to the energy dissipated during the reversible domain wall bulging process, whereas for increasing $h_{\mathrm{ac}}$ such peak remains, but the additional low-frequency peak corresponding to the hysteretic mechanism appears. The height of hysteretic peak increases as $h_{\mathrm{ac}}$ increases, because the higher the field, the easier DWs displacement, for which larger energy dissipation occurs (compared with domain bulging) leading to the observed higher peaks for increasing $h_{\text {ac }}$ fields.

Figure 11. Spectroscopic plots $\mu_{\mathrm{im}}(f)$ for glass coated $\mathrm{Co}_{69.7} \mathrm{Fe}_{3.8} \mathrm{~B}_{13} \mathrm{Si}_{11} \mathrm{Mo}_{1.5} \mathrm{Ni}_{1}$ microwire at various $h_{\mathrm{ac}}$ field values.

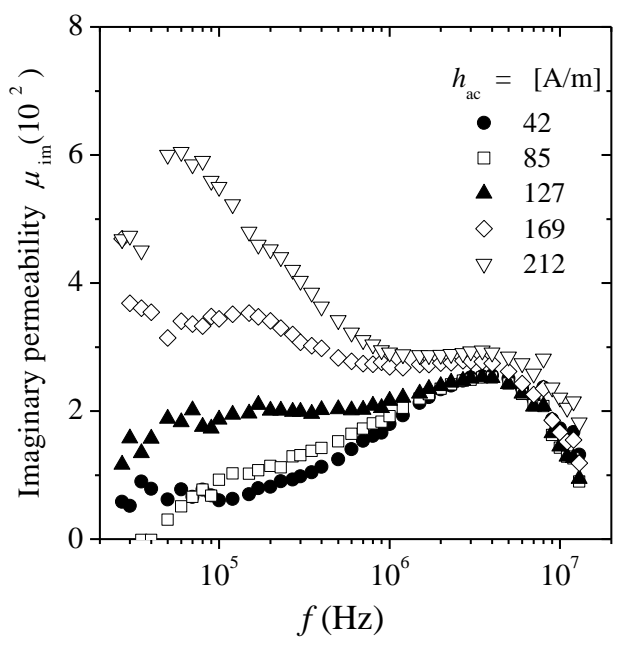


Circular magnetization curves $\mathrm{M}_{\phi}\left(h_{a c}\right)$ at two selected frequencies are shown in Figure 12. The low frequency $(f=20 \mathrm{kHz})$ curve exhibits the expected features of domain wall magnetization, such as a rather low initial susceptibility (for $h_{a c}$ below $100 \mathrm{~A} / \mathrm{m}$ ) followed by a marked increase in slope at about $h_{a \mathrm{c}}=100 \mathrm{~A} / \mathrm{m}$, together with a trend to saturation above $200 \mathrm{~A} / \mathrm{m}$. In contrast, the curve obtained at $10 \mathrm{MHz}$ (high frequency) shows a linear tendency in agreement with a pure rotational magnetization process.

Figure 12. Circular magnetization curves for glass coated $\mathrm{Co}_{69.7} \mathrm{Fe}_{3.8} \mathrm{~B}_{13} \mathrm{Si}_{11} \mathrm{Mo}_{1.5} \mathrm{Ni}_{1}$ microwire at low $\left(f<<f_{\mathrm{x}}\right)$ and high $\left(f>>f_{\mathrm{x}}\right)$ frequency values.

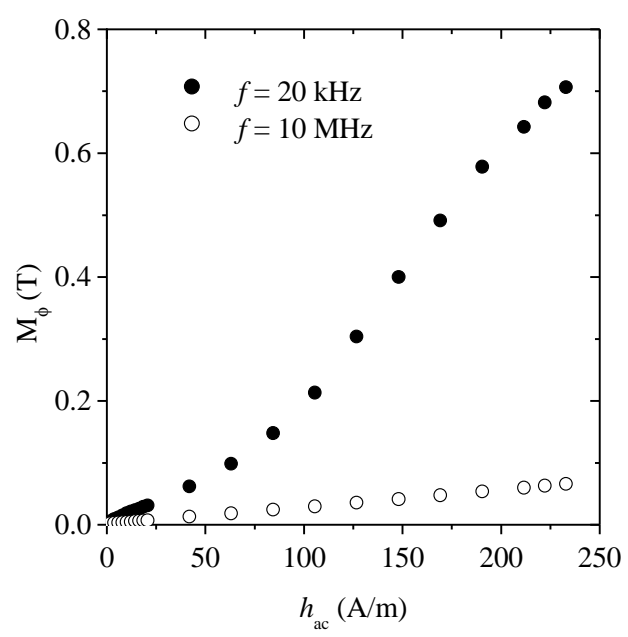

The Cole-Cole representation for the reversible bulging of DWs and the hysteresis process for the same glass coated $\mathrm{Co}_{69.7} \mathrm{Fe}_{3.8} \mathrm{~B}_{13} \mathrm{Si}_{11} \mathrm{Mo}_{1.5} \mathrm{Ni}_{1}$ microwire is depicted in Figure 13 for two distinct $\mathrm{h}_{\mathrm{ac}}$ amplitudes of $42 \mathrm{~A} / \mathrm{m}$ and $212 \mathrm{~A} / \mathrm{m}$. A well defined semicircle formation (each point obtained at a different frequency) is observed for $h_{\mathrm{ac}}=42 \mathrm{~A} / \mathrm{m}$ with a diameter of $7.5 \mu \mathrm{H}$ associated to the former magnetization mechanism, whilst for $h_{\mathrm{ac}}=212 \mathrm{~A} / \mathrm{m}$, two half-circles of different diameter are evident, the larger one being of $15 \mu \mathrm{H}$ diameter and for which the irreversible displacement of DWs is attributed.

Figure 13. Cole-Cole plots at different $h_{a c}$ amplitudes for glass coated $\mathrm{Co}_{69.7} \mathrm{Fe}_{3.8} \mathrm{~B}_{13} \mathrm{Si}_{11} \mathrm{Mo}_{1.5} \mathrm{Ni}_{1}$ microwire.

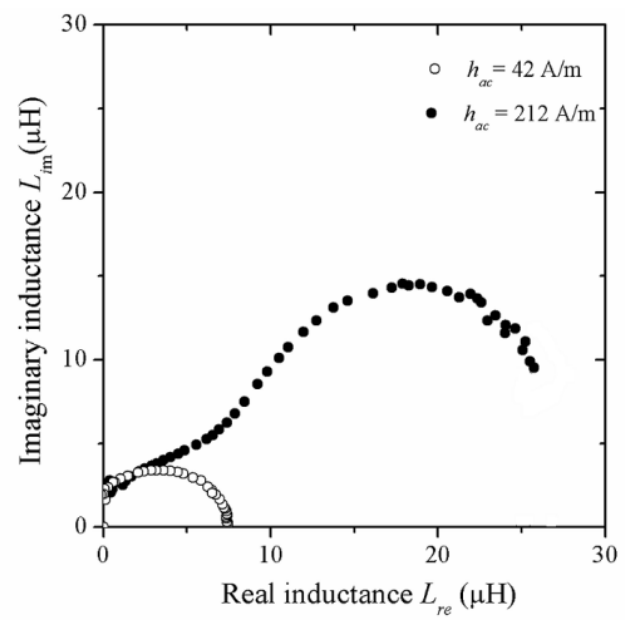




\subsection{Biphase Wires}

Recently, amorphous glass coated microwires have elicited considerable interest for the development of biphase microwires consisting of an amorphous soft magnetic metallic nucleus (obtained by Taylor-Ulitovsky technique) surrounded by a hard magnetic layer deposited by electrochemical process on the glass layer [41]. This hard magnetic layer affords the control of the biphase microwires magnetic properties by means of its magnetic interaction with the soft magnetic nucleus through: (i) the modification of the amorphous wire easy axis of magnetization from radial to longitudinal orientation depending on the hard layer thickness, (i.e., on the electrochemical deposition parameters, namely current density and electrochemical exposure time); (ii) the possibility for obtaining controllable magnetostatic or eventually exchange bias response (similar to the bias effect used in spin valves); and (iii) the tailoring of their magnetic domain formation and thus, their magnetic performance from non-hysteretic to bistable behavior [42]. However, biphase systems entailing an amorphous wire nucleus (obtained by rotating water bath melt spinning method) have not yet been explored at all. The spectroscopy response for real and imaginary components of complex permeability for biphase wires composed of an amorphous wire nucleus of composition $\mathrm{Fe}_{64} \mathrm{Co}_{8} \mathrm{~B}_{19.2} \mathrm{Si}_{4.8} \mathrm{Ta}_{4}$ (140 $\mu$ m diameter) and electrodeposited $\mathrm{Co}_{90} \mathrm{Ni}_{10}$ magnetic hard layers with variable thickness $t$ of $1.5 \mu \mathrm{m}, 3 \mu \mathrm{m}$ and $6 \mu \mathrm{m}$ (attained by carefully control of exposure time to the electrodeposition process) is shown in Figure 14. For the $\mu_{\mathrm{re}}(f)$ plot of $t=0 \mu \mathrm{m}$ (Figure 14a), the reversible bulging of the magnetic DWs is manifested for increasing frequency $f$ values up to $10^{3} \mathrm{~Hz}$. This part of the real permeability curve corresponds to the initial permeability $\mu_{\text {ini }}$ of the soft magnetic amorphous wire core. Further increase in $f$ leads to relaxation of the reversible bulging mechanism at $f=9 \mathrm{kHz}$, leaving the spin rotation as the only magnetization process active for $f>f_{\mathrm{x}}$. The hard layer thickness significantly affects the low-field reversible magnetization process of the soft core since $\mu_{\text {ini }}$ decreases from $16,738(t=0)$ to $3,205(t=6 \mu \mathrm{m})$. This marked reduction for $\mu_{\text {ini }}$ can be attributed to the increasing out-of-axis anisotropy, developed by the biphase wires with increasing hard layer thickness, as a consequence of the accumulation of mechanical stress appearing at the amorphous-wire/CoNi-layer interface, for which the thicker the layer, the higher the internal stress accumulated.

Alternatively, the imaginary $\mu_{\text {im }}$ component (Figure 14b) is associated with the magnetic losses (hysteresis, eddy current or power losses) [25]. Consequently, the presence of the external hard layer significantly reduces the losses. The maximum in $\mu_{\mathrm{im}}$ is observed at frequency $f_{\mathrm{x}}$ for each biphase wire. The $f_{\mathrm{x}}$ exhibits an increasing tendency (Figure 14b) from $9 \mathrm{kHz}(t=0 \mu \mathrm{m})$ to $27 \mathrm{kHz}(t=6 \mu \mathrm{m})$ and follows an inverse tendency with $t$, which confirms the influence of the variable anisotropy on the spin rotation mechanism active for $f>f_{\mathrm{x}}$, for which the product $\mu_{\mathrm{re}} f_{\mathrm{x}}=$ constant is known as the Snoek's limit [26]. 
Figure 14. Real and imaginary permeability spectra for biphase wires with hard layer thickness $t$ (microns) as a parameter.

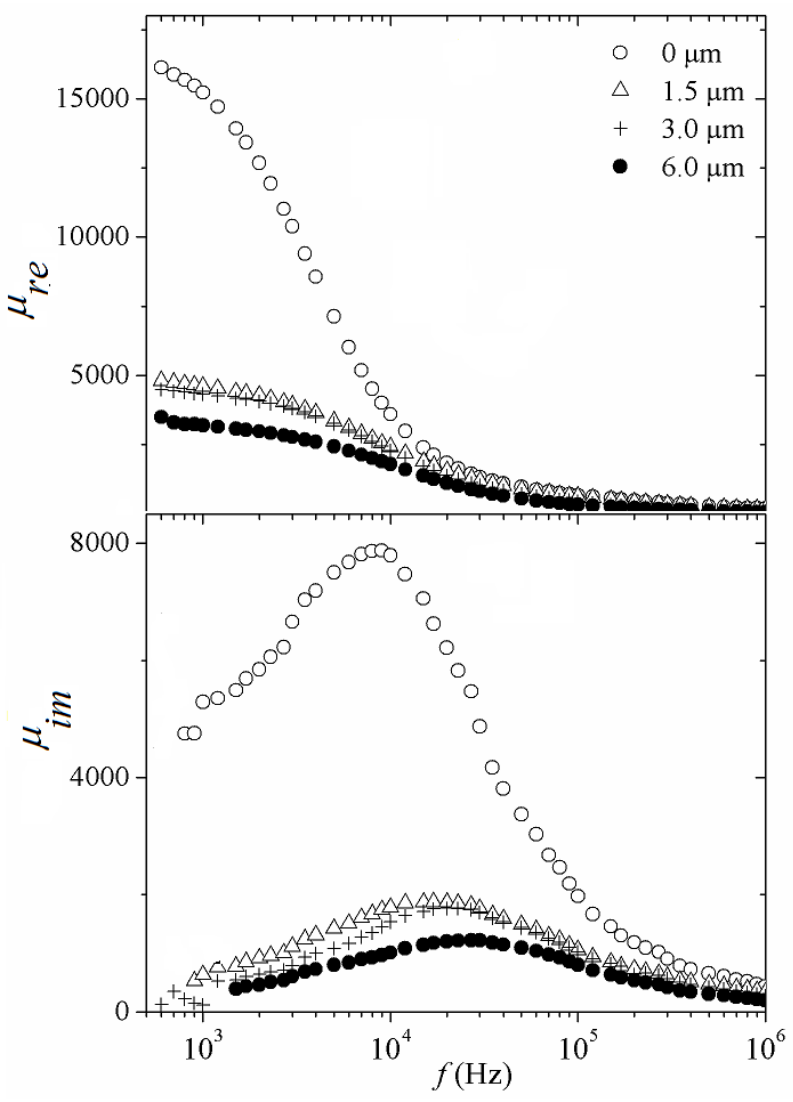

\section{Experimental Section}

Melt spun ribbons with compositions $\mathrm{Fe}_{80} \mathrm{~B}_{10} \mathrm{Si}_{10-\mathrm{x}} \mathrm{Ge}_{\mathrm{x}}$ and $\mathrm{Fe}_{52-\mathrm{x}} \mathrm{Co}_{10+\mathrm{x}} \mathrm{Nb}_{8} \mathrm{~B}_{30}$ were prepared by means of chill block melt spinning technique with a roll speed of $35 \mathrm{~m} / \mathrm{s}$, from initial ingots obtained from arc-melting of pure $(99.99 \%)$ constituent elements in a Ti-gettered, protective Ar atmosphere. On the other hand, as-cast amorphous $\left(\mathrm{Co}_{0.94} \mathrm{Fe}_{0.6}\right)_{72.5} \mathrm{~B}_{15} \mathrm{Si}_{12.5}$ wires of $120 \mu \mathrm{m}$ diameter were prepared by the rotating water bath melt spinning method by using a water depth of $19 \mathrm{~mm}$ and an angular drum velocity of $370 \mathrm{rpm}$. Additionally, amorphous glass coated microwires, $25 \mu \mathrm{m}$ metallic diameter and $10 \mu \mathrm{m}$ glass coating of nominal composition $\mathrm{Co}_{69.7} \mathrm{Fe}_{3.8} \mathrm{~B}_{13} \mathrm{Si}_{11} \mathrm{Mo}_{1.5} \mathrm{Ni}_{1}$ were prepared by the Taylor-Ulitovsky technique.

For impedance measurements, an HP 4192 A impedance analyzer controlled by a PC was used. Our measuring system allows the determination of the complex impedance for 94 discrete frequencies from $10 \mathrm{~Hz}$ to $13 \mathrm{MHz}$ together with the rms voltage varying between 0.1 and $1.0 \mathrm{~V}$, which leads to longitudinal ac applied magnetic fields $h_{\mathrm{ac}}$ within the range $0.40-3 \mathrm{~A} / \mathrm{m}$ on amorphous ribbons by means of a coil with 100 turns; whereas for amorphous wires and microwires, the rms voltage applied directly on the wire ends afforded ac currents $i_{a c}$ flowing through the sample within the range 2-22 mA (rms) which, in turn, produce circular $h_{\mathrm{ac}}$ fields within the range 10-240 A/m. 


\section{Conclusions}

Inductance spectroscopy has been employed as a powerful tool for characterization of the magnetization dynamics in amorphous ribbons and wires, by means of the resolution of the magnetization mechanism within the materials, in terms of reversible deformation of pinned domain walls, domain wall displacements and spin rotation, for which characteristic parameters, such as the alloy initial permeability and the relaxation frequencies, indicating the dispersion of each process, can be established. In the case of amorphous ribbons, the reversible bulging of DWs and its further displacement was observed as being dependent on the intensity of the ac applied field, together with a clear dependence of both the magnetic permeability and the relaxation frequency with the chemical composition in FeBSiGe alloys. Further, combined experiments with magnetic dc fields and tensile stress, afforded the estimation of the magnetostriction constant in FeCoNbB ribbons. On the other hand, for amorphous wires of 120 microns diameter, the calculation of their circular magnetization curves also reflect the frequency dependency of the magnetization mechanisms identified, as well as the effect of induced anisotropies (by means of torsion stress). In addition, amorphous microwires exhibited similar features for their magnetization mechanism resolution, with distinctive higher relaxation frequencies (compared with amorphous wires) for both reversible bulging of DWs and their displacement, because of the reduced diameters characterizing these alloys obtained by means of the Taylor-Ulitovsky method. Finally, the typical inverse correlation between the alloy's initial permeability and its frequency relaxation was verified for biphase wires, for which the variable hard coating thickness also influences the magnetic response.

\section{Acknowledgements}

The author is grateful with M.Vazquez (Materials Science Institute, CSIC, Madrid, Spain) for his kindly supply of microwires and biphase samples.

\section{References}

1. Hasegawa, R. Present status of amorphous soft magnetic alloys. J. Magn. Magn. Mater 2000, 215/216, 240-245.

2. Gavrila, H.; Ionita, V. Crystalline and amorphous soft magnetic materials and their applications status of art and challenges. J. Optoelectronic Adv. Mater. 2002, 4, 173-192.

3. Kavesh, S. Metallic Glasses; The American Society for Metals: Ohio, OH, USA, 1978; p. 36.

4. Masumoto, T.; Ohnaka, I.; Inoue, A.; Hadiwara, M. Production of Pd-Cu-Si amorphous wires by melt spinning method using rotating water. Scripta Metall. 1981, 15, 293-298.

5. Vazquez, M. Soft magnetic wires. Physica B 2001, 299, 302-313.

6. Macdonald, R. Impedance Spectroscopy; Wiley\&Sons: New York, NY, USA, 1987.

7. Valenzuela, R. Impedance spectroscopy in ferromagnetic materials. Mat. Res. Soc. Symp. Proc. 2002, 699, 127-133.

8. Irvine, J.T.S.; Amano, E.; Huanosta, A.; Valenzuela, R.; West, A.R. Characterisation of magnetic materials by impedance spectroscopy. Sol. State Ionics 1990, 40/41, 220-223. 
9. Amano, E.; Valenzuela, R.; Irvine, J.T.S.; West, A.R. Domain wall relaxation in amorphous ribbons. J. Appl. Phys. 1990, 67, 5589-5591.

10. Quintana, P.; Amano, E.; Echavarrieta, C.; Valenzuela, R.; González, J.; Murillo, N.; Blanco, J.M. Effect of tensile stress on the domain wall dynamics of co-based amorphous ferromagnetic wires. J. Magn. Magn. Mater. 1995, 140-144, 1903-1904.

11. Montiel, H.; Alvarez, G.; Gutiérrez, M.P.; Zamorano, R.; Valenzuela, R. The effect of metal-to-glass ratio on the low field microwave absorption at $9.4 \mathrm{ghz}$ of glass coated cofebsi microwires. IEEE Trans. Magn. 2006, 42, 3380-3382.

12. Sanchez, M.L.; Valenzuela, R.; Vazquez, M.; Hernando, A. Circumferential permeability in non-magnetostrictive amorphous wires. J. Mater. Res. 1996, 11, 2486-2489.

13. Kronmüller, H.; Fernengel, W. The role of internal stresses in amorphous ferromagnetic alloys. Phys. Stat. Sol. A 1981, 64, 593-602.

14. Vazquez, M.; Fernengel, W.; Kronmüller, H. The effect of tensile stresses on the magnetic properties of $\mathrm{Co}_{58} \mathrm{Fe}_{5} \mathrm{Ni}_{10} \mathrm{Si}_{11} \mathrm{~B}_{16}$ amorphous alloys. Phys.Stat. Sol. A 1983, 80, 195-204.

15. Appino, C.; Fiorillo, F.; Maraner, A. Initial susceptibility vs. applied stress in amorphous alloys with positive and negative magnetostriction. IEEE Trans. Magn. 1993, 29, 3469-3471.

16. Schäfer, R. Domains in "Extremely" soft magnetic materials. J. Magn. Magn. Mater. 2000, 215-216, 652-663.

17. Zhukov, A.P.; Vázquez, M.; Velázquez, J.; Chiriac, H.; Larin, V. The remagnetization process in thin and ultra-thin Fe-rich amorphous wires. J. Magn. Magn. Mater. 1995, 151, 132-138.

18. Hernando, A.; Marín, P. Magnetic microwires: Manufacture, properties and applications. In Concise Encyclopedia of Magnetic and Superconducting Materials, 2nd ed.; Buschow, K.H.J., Ed.; Elsevier: Amsterdam, The Netherlands, 2005; p. 511.

19. Chiriac, H.; Óvári, T.A.; Pop, G. Internal stress distribution in glass-covered amorphous magnetic wires. Phys. Rev.B 1995, 52, 10104-10113.

20. Velázquez, J.; Vázquez, M.; Zhukov, A.P. Magnetoelastic anisotropy distribution in glass-coated microwires. J. Mater. Res. 1996, 11, 2499-2505.

21. Vazquez, M. Advanced magnetic microwires. In Handbook of Magnetism and Advanced Magnetic Materials; Kronmüller, H., Parking, S., Eds.; Wiley: Chichester, UK, 2007; p. 2193.

22. Valenzuela, R. Low-frequency magnetoimpedance: Domain wall magnetization processes. Physica B 2001, 299, 280-285.

23. Valenzuela, R.; Montiel, H.; Gutiérrez, M.P.; Betancourt, I. Characterization of soft ferromagnetic materials by inductance spectroscopy and magnetoimpedance. J. Magn. Magn. Mater. 2005, 294, 239-244.

24. Valenzuela, R. Giant magnetoimpedance and inductance spectroscopy. J. Alloys Compounds 2004, 369, 40-42.

25. Chen, D.X.; Muñoz, J.L. AC impedance and circular permeability of slab and cylinder. IEEE Trans. Magn. 1999, 35, 1906-1923.

26. Chikazumi, S. Physics of Magnetism; Robert, E., Ed.; Krieger Pub Co: New York, NY, USA, 1978.

27. McHenry, M.E.; Willard, M.A.; Laughlin, D.E. Amorphous and nanocrystalline materials for applications as soft magnets. Prog. Mater. Sci. 1999, 44, 291-433. 
28. Fdez-Gubieda, M.L.; García-Arribas, A.; Barandiarán, J.M.; López Antón, R.; Orue, I.; Gorria, P.; Pizzini, S.; Fontaine, A. Local structure and ferromagnetic character of Fe-B and Fe-B-Si amorphous alloys. Phys. Rev. B 2000, 62, 5746-5752.

29. Barandiarán, J.M.; Gorria, P.; Orúe, I.; Fdez-Gubieda, M.L.; Plazaola, F.; Hernando, A. Tensile stress dependence of the curie temperature and hyperfine field in $\mathrm{Fe}-\mathrm{Zr}-\mathrm{B}-(\mathrm{Cu})$ amorphous alloys. Phys. Rev. B 1996, 54, 3026-3029.

30. García, K.L.; Valenzuela, R. The effects of the axial DC field on magnetoimpedance: Circumferential domain wall damping. IEEE Trans. Magn. 1998, 34, 1162-1164.

31. Valenzuela, R.; Irvine, J.T.S. Domain wall relaxation frequency and magnetocrystalline anisotropy constant in Ni-Zn ferrites. J. Magn. Magn. Mater. 1996, 160, 386-387.

32. Panina, L.V.; Mohri, K.; Uchiyama, T.; Noda, M. Giant magnetoimpedance in co-rich amorphous wires and films. IEEE Trans. Magn. 2002, 31, 1249-1260.

33. Knobel, M.; Vazquez, M.; Kraus, L.; Magneotimpedance, G. Handbook of Magnetic Materials; Buschow, K.H.J., Ed.; Elsevier North-Holland: Amsterdam, The Netherlands, 2003; Volume 15, p. 497.

34. Hernando, A.; Vazquez, M.; Madurga, V.; Kronmüller, H. Modification of the saturation magnetostriction constant after thermal treatments for the $\mathrm{Co}_{58} \mathrm{Fe}_{5} \mathrm{Ni}_{10} \mathrm{~B}_{16} \mathrm{Si}_{11}$ amorphous ribbons. J. Magn. Magn. Mater. 1983, 37, 161-166.

35. Madurga, V.; Hernando, A. Radial stress distribution generated during rapid solidification of amorphous wires. J. Phys. Cond. Mater. 1990, 2, 2127, doi:10.1088/0953-8984/2/9/002.

36. Tannous, C.; Gieraltowski, J.; Valenzuela, R. Helical anisotropy and magnetoimpedance of CoFeSiB wires under torsional stress. J. Magn. Magn. Mater. 2007, 308, 273-277.

37. Garcia, K.L.; Garcia-Beneytez, J.M.; Valenzuela, R.; Zhukov, A.; Gonzalez, J.; Vazquez, M. Effects of torsion on the magnetoimpedance response of CoFeBSi amorphous wires. J. Magn. Magn. Mater. 2001, 226/230, 721-723.

38. Chubykalo, O.A.; Gonzalez, J.; Gonzalez, J.M. Saturation magnetostriction dependence on torsion in amorphous wires measured by modified small angle magnetization rotation method. J. Magn. Magn. Mater. 1997, 169, 169-177.

39. Liu, J.; Malmhall, R.; Arnberg, L.; Savage, S.J. Theoretical analysis of residual stress effects on the magnetostrictive properties of amorphous wires. J. Appl. Phys. 1990, 67, 4238-4240.

40. Kim, C.G.; Yoon, S.S.; Vazquez, M. Evaluation of helical magnetoelastic anisotropy in fe-based amorphous wire from the decomposed susceptibility spectra. J. Magn. Magn. Mater. 2001, 223, 199-202.

41. Pirota, K.R.; Provencio, M.; Garcia, K.L.; Escobar-Galindo, R.P.; Zelis, M.; Hernández-Vélez, M.; Vazquez, M. Bi-magnetic wires: A novel family of materials with controlled magnetic behavior. J. Magn. Magn. Mater. 2005, 68, 290-291.

42. Vázquez, M.; Castaño, F.; Ovari, T.A.; Raposo, V.; Hernando, A. New viscosimeter based on the AC field induced rotation of magnetostrictive amorphous wires. Sensor. Actuator. A-Phys. 2001, 91, 112-115.

(C) 2010 by the authors; licensee MDPI, Basel, Switzerland. This article is an open access article distributed under the terms and conditions of the Creative Commons Attribution license (http://creativecommons.org/licenses/by/3.0/). 